\title{
Evolution of coauthorship networks: worldwide scientific production on leishmaniasis
}

\author{
Gregorio González-Alcaide ${ }^{[1]}$, Charles Huamani ${ }^{[2]}$, Jinseo Park ${ }^{[3]}$ and José Manuel Ramos ${ }^{[4]}$
}

[1]. Department of History of Science and Documentation, University of Valencia, Valencia, Spain. [2]. Instituto Nacional de Salud, Lima, Peru. [3]. Korea Institute of Science \& Technology Information, Deajeon, Korea. [4]. Department of Internal Medicine, Hospital General Universitario de Alicante, Alicante, Spain.

\begin{abstract}
Introduction: Collaboration is one of the defining features of contemporary scientific research, and it is particularly important with regard to neglected diseases that primarily affect developing countries. Methods: The present study has identified publications on leishmaniasis in the Medline database from 1945 to 2010, analyzing them according to bibliometric indicators and statistics from social network analysis. Examining aspects such as scientific production, diachronic evolution, and collaboration and configuration of the research groups in the field, we have considered the different types of Leishmania studied and the institutional affiliation and nationality of the authors. Results: Seven-hundred and thirty-five authors participate in 154 prominent research clusters or groups. Although the most predominant and consolidated collaborations are characterized by members from the same country studying the same type of Leishmania, there are also notable links between authors from different countries or who study different clinical strains of the disease. Brazil took the lead in this research, with numerous Brazilian researchers heading different clusters in the center of the collaboration network. Investigators from the USA, India, and European countries, such as France, Spain, the United Kingdom, and Italy, also stand out within the network. Conclusions: Research should be fostered in countries such as Bangladesh, Nepal, Sudan, and Ethiopia, where there is a high prevalence of different forms of the disease but limited research development with reference authors integrated into the collaboration networks.
\end{abstract}

Keywords: Leishmaniasis. Social Network Analysis. Coauthorship network. Scientific collaboration.

Bibliometrics. Scientific network evolution.

\section{INTRODUCTION}

Leishmaniasis is a parasitic zoonosis estimated to affect 15 million people worldwide, with nearly two million new cases reported every year. Despite these figures, leishmaniasis is also considered a neglected disease ${ }^{1}$. The World Health Organization (WHO) has promoted different initiatives to foster the development of new methods of prevention, diagnosis, and treatment ${ }^{2}$; scientific collaboration is an essential part of that process, particularly in developing countries in which there are few resources dedicated to research ${ }^{3}$.

Since the early $20^{\text {th }}$ century, bibliometrics has been shaped as a discipline that uses the quantitative analysis of scientific publications to evaluate the size, growth, and distribution of scientific bibliography. Similarly, the field examines scientific agents that produce, transmit, and use the body of research, analyzing their visibility and impact in subsequent literature ${ }^{4}$. $\mathrm{Smith}^{5}$ reviewed the main bibliometric studies that have analyzed the area of tropical medicine throughout the past several decades;

Address to: Dr. Gregorio González-Alcaide. Faculty of Medicine and Odontology/ Department of History of Science and Documentation/University of Valencia. 15, Blasco Ibáñez Avenue, 46010 Valencia, Spain.

Phone: 3496 386-4958; Fax: 3496 661-6756

e-mail: gregorio.gonzalez@uv.es

Received 7 October 2013

Accepted 3 December 2013 these studies are generally focused on the study of scientific production, citation, or the impact factor of the journals in the field. Recently, different papers have examined the scientific production specific to leishmaniasis ${ }^{6-8}$. All of these studies report an increase in both scientific production on leishmaniasis and collaboration among institutions and countries. However, the collaboration among authors and the formation of research groups in the field have not been evaluated, and no studies have analyzed whether research collaboration is primarily based on the study of the same type or of different clinical strains of leishmaniasis (where different interests would exist to obtain results for the control and management of the disease). In this sense, the study of scientific coauthorship through social network analysis allows for a more precise analysis of the collaboration structures within a discipline or area of knowledge than studies based on bibliometric indicators alone.

The aim of the present study is to analyze collaboration among researchers with regard to the global scientific production on leishmaniasis considering the different forms of the disease and studying the diachronic evolution of the formation of research groups in the field.

\section{METHODS}

The study was carried out via a bibliographic search on the PubMed platform, which was used to identify indexed documents with the keywords or medical subject headings (MeSH) 
Leishmania and leishmaniasis published between 1945 and 2010. The bibliographic information from the collected registries was introduced into a locally stored, relational database to carry out a bibliographic normalization or homogenization of data and the quantitative analysis. The normalization process was conducted to consolidate variations of author names. Such consolidation is often necessary because an author may be identified by one first name or more than one, initials, or a full name or due to the use of hyphens to separate names. The criterion followed in this homogenization process was the occurrence of the institutional signature associated with the variations of names and surnames.

We identified the primary form of Leishmania studied by researchers by quantifying the MeSH descriptors assigned to the documents as well as their country. The authors were assigned to the form of Leishmania cited most frequently as a MeSH term in the documents published (cutaneous leishmaniasis, diffuse cutaneous leishmaniasis, and visceral leishmaniasis).

With the information obtained, we carried out a social network analysis, applying it to the coauthorships of the scientific publications and using the documents published on the Medline database as the unit of analysis. Bibexcel software (developed by Olle Persson, Inforsk, Umeã University, Sweden) was used to process the bibliographic information ${ }^{9}$, whereas the statistical calculations and visual representation of the networks were performed with the social network analysis program Pajek ${ }^{10}$.

Below, we delineate the indicators and concepts used in the present study.

\section{Collaboration index}

A widely used bibliometric indicator that establishes the average number of authors who have participated in the group of analyzed documents.

\section{Network}

A graphic representation (graph) comprised of a group of nodes or vertices (authors of scientific publications) and links between the nodes, which represent coauthorships or joint signatures from one or more scientific publications.

\section{Network average degree}

The average number of collaborators per author.

\section{Collaboration threshold}

The degree of intensity of the links established between the nodes. The threshold or collaboration intensity is higher for a greater number of papers signed by two given authors. Graphically, the collaboration threshold has been expressed in the networks using a thicker line representing the links.

\section{Component}

A group of nodes that are interconnected directly or through intermediaries.

\section{Size of the largest component or giant component}

Number of interconnected authors that comprise the largest component, considering all links and without applying any collaboration threshold.

\section{Isolated researchers}

Researchers who are not connected with any other researcher.

\section{Network density}

The proportion between the number of links established in the network and the maximum number of links that are theoretically possible.

\section{Average distance}

The average number of intermediaries between linked nodes.

\section{Research clusters or research groups}

Scientists working together in partnership to realize shared goals. In the present study, these clusters were identified by applying a high collaboration threshold to identify the stable and consolidated collaboration relationships, that is, the groups of authors that maintain a higher degree of interconnectedness and therefore cohesion and differentiation compared to other groups.

\section{Cutpoint or articulation point}

Nodes through which all paths or geodesics pass, allowing linkage between other nodes; if these points were eliminated, parts of the network would become disconnected, increasing the number of components and reducing their size. Thus, these authors play an essential role (key players) as intermediaries in ensuring connectivity and communication between different parts of the network, preventing them from becoming isolated.

\section{RESULTS}

We identified 20,780 documents on leishmaniasis published between 1945 and 2010, signed by 35,588 authors. The diachronic evolution displays constant growth in the number of documents published and researchers who have participated in studies on leishmaniasis (Table 1).

With regard to the collaboration indicators, we observed sustained increases in both the mean number of signatures on the papers and the average number of author collaborators (Figure 1).

The social network analysis statistics from the coauthorship network of scientific publications on leishmaniasis indicate that the size of the largest component has gradually increased according to the increase in the number of researchers that comprise the network. The relationships maintained between authors have also multiplied, with $80.9 \%$ of all authors integrated in the largest component in the 2001-2010 period. Inversely, the number of isolates has been progressively decreasing, falling to less than $1 \%$ in the last decade studied. The network density has also declined as its size has increased. Finally, the average distance between the researchers making up the network grew progressively, reaching 6.01 in 1981-1990, before decreasing slightly in the last two decades (Table 1).

The scientific production and number of collaborators of the 25 most productive authors ( $>99$ papers) are also shown (Table 2). Most of the group ( $\mathrm{n}=17)$ initiated their scientific 
TABLE 1 - Indicators of production and collaboration in scientific publications on leishmaniasis (1945-2010).

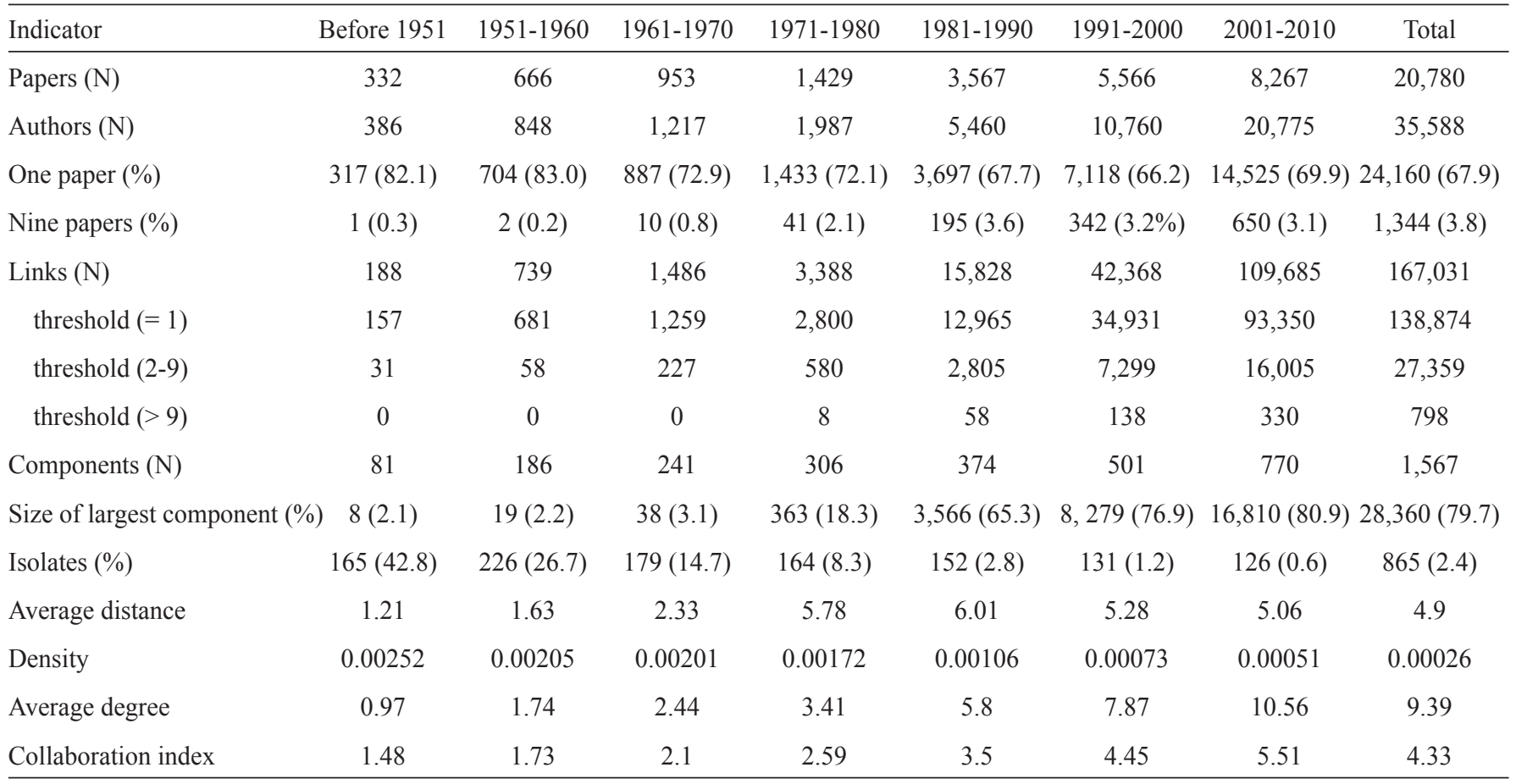

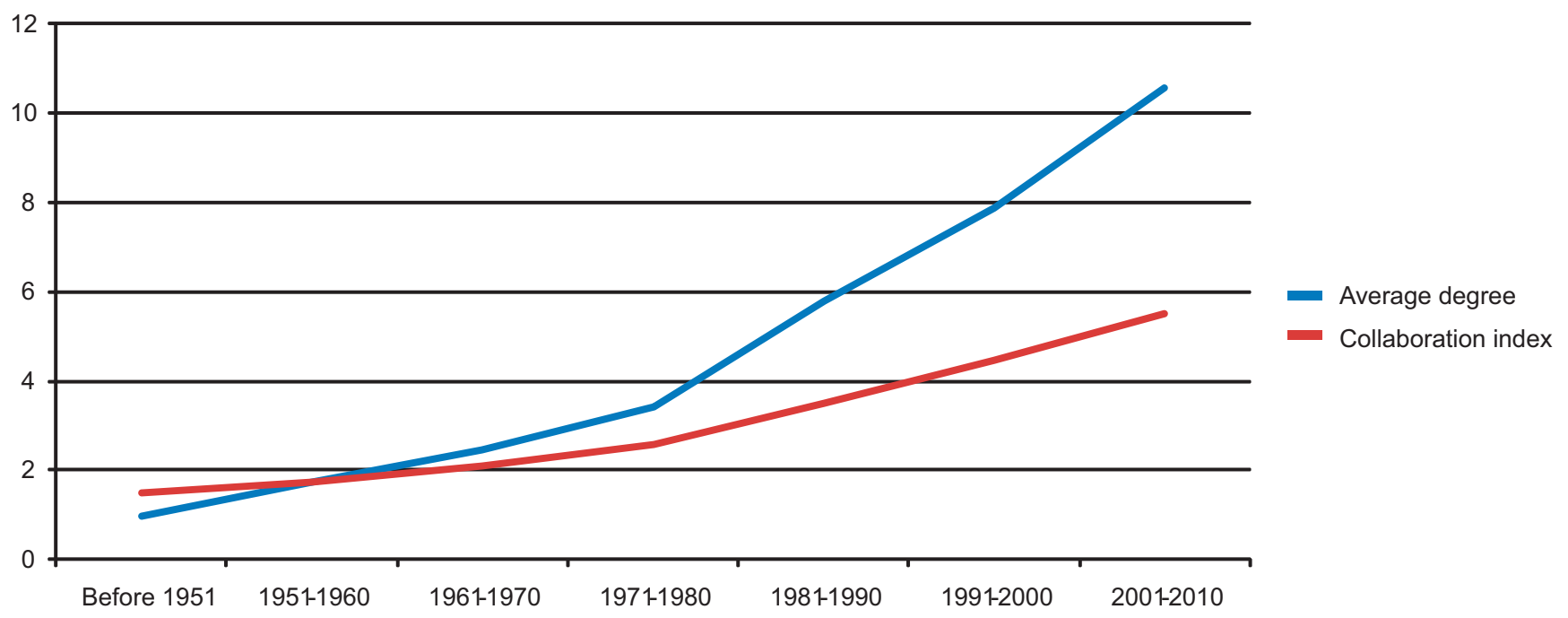

FIGURE 1 - Evolution by decade of the collaboration index and the average degree in publications on leishmaniasis.

activity prior to the $1981-1990$ period, whereas the remainder $(\mathrm{n}=8)$ did so during that period, and all have remained active until the present. In contrast, some researchers have shown a decline in scientific production in the most recent period. All researchers have had sporadic collaborations with a high number of authors, but they only have a small group of regular collaborators with whom they publish a significant proportion of their scientific production. In that sense, the 25 most productive authors have published 10 or more papers with only $0.7 \%-8.97 \%$ of their total collaborators.

We have identified 1,567 components comprised of two or more authors who are connected by at least one coauthorship. Seven-hundred and thirty-five authors comprise 154 research clusters, which constitute important research foci whose authors maintain close relationships with each other; indeed, the links between the members are derived from the fact that they have 


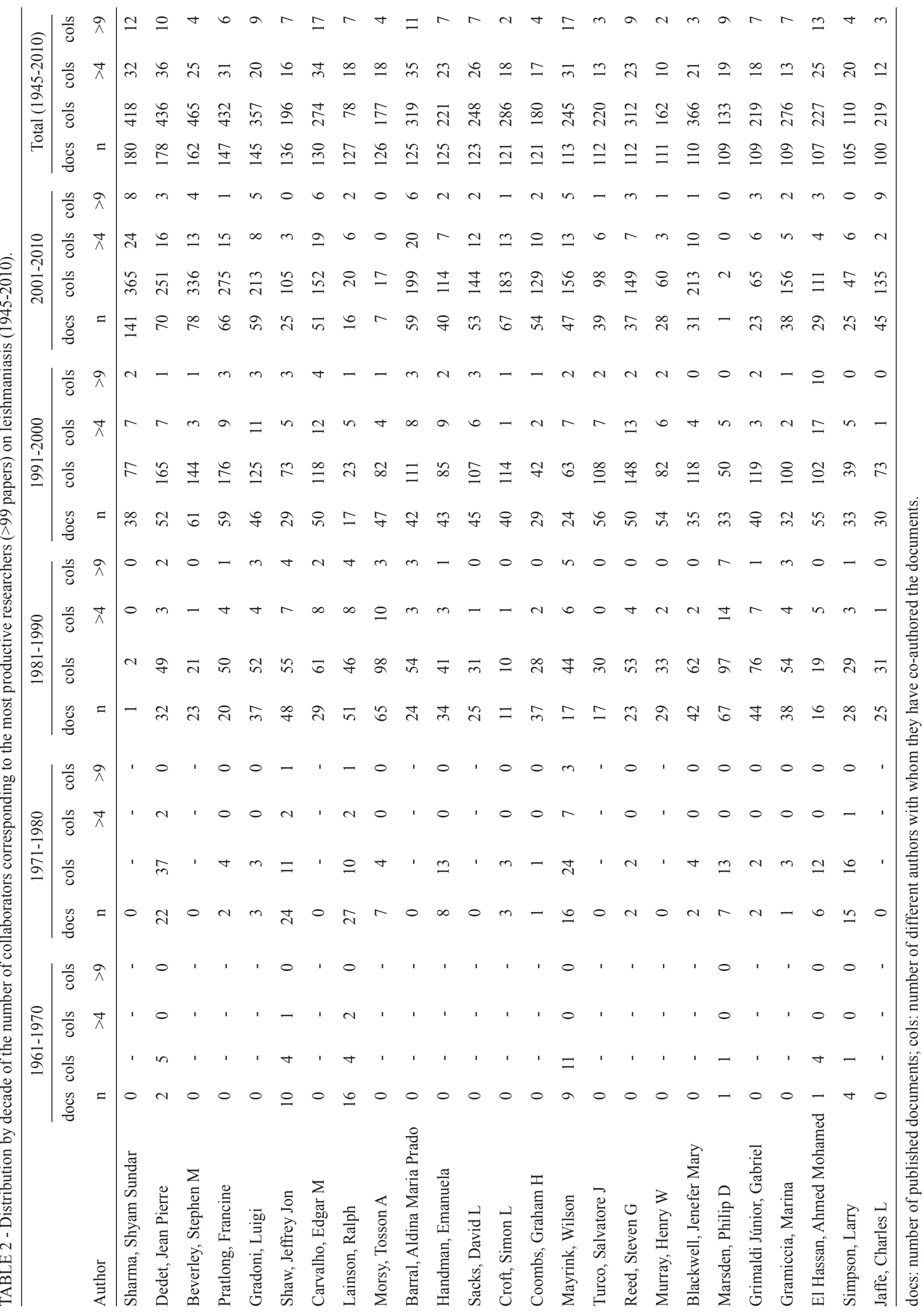


coauthored at least 10 papers. The visual representation of these clusters (Figure 2) illustrates the rank that the 101 most productive authors ( $>49$ publications) hold as well as the type of leishmaniasis that they normally study and their country of origin. The largest cluster has 85 authors. The five clusters that follow in size bring together 36, 26, 20, 19, and 18 authors. Eight others comprise 10-14 authors, whereas the remaining 140 have two to nine authors. Most of the 101 most productive authors occupy a prominent position in the clusters in which they participate, drawing together other less productive authors or serving as important cutpoints between different groups of authors.

The 735 authors identified belong to 49 countries; Brazilians predominate $(\mathrm{n}=140)$, followed by American $(\mathrm{n}=105)$, French $(\mathrm{n}=72)$, Indian $(\mathrm{n}=55)$, Spanish $(\mathrm{n}=44)$, British $\mathrm{n}=38)$, Italian $(n=25)$, Swiss $(n=24)$, and German $(n=20)$ authors. In general, the predominant links in the clusters are between authors who study the same type of leishmaniasis and are from the same country. However, some inter-Leishmania connections stand out. In terms of international collaborations, the links that some American researchers have with Brazilian and Indian colleagues are also worth noting.

At an institutional level, three Brazilian centers stand out as having the greatest number of affiliated authors on the subject of leishmaniasis: the Oswaldo Cruz Foundation $(n=45)$, the Federal University of Bahia ( $\mathrm{n}=15)$, and the University of São Paulo ( $\mathrm{n}=12$ ).
The next positions are held by the Rajendra Memorial Research Institute of Medical Sciences of India $(\mathrm{n}=11)$ and the Tehran University of Medical Sciences of Iran ( $n=10)$. In the West, University of Montpellier of France $(n=8)$ and Ohio State University of the USA $(n=7)$ lead the ranking. The latter of these countries is characterized by highly dispersed scientific production among its universities, with 105 authors who are distributed among 67 different institutions, as opposed to the 36, 33, and 23 centers identified in France, Brazil, and India, respectively.

In the same way that research groups tend to be clustered in a single country, many authors of the same cluster belong to a single institution. However, there are outstanding collaboration links between the main institutions involved in tropical diseases research and training (e.g., in the largest cluster between the Federal University of Bahia and the University of Brasilia in Brazil; the CSIR-Central Drug Research Institute and the Balaji Utthan Sansthan in India; and the Institute of Tropical Medicine and the University of Antwerp in Belgium).

With regard to the formation of the research clusters, we devised a graphic representation (Figure 3) that illustrates the evolution by decade (1981-2010) of the authors belonging to the largest component from the 2001-2010 period. Applying a collaboration threshold of four or more coauthorships, we also indicate the position occupied by the 25 most productive authors to facilitate the visualization of the networks and focus

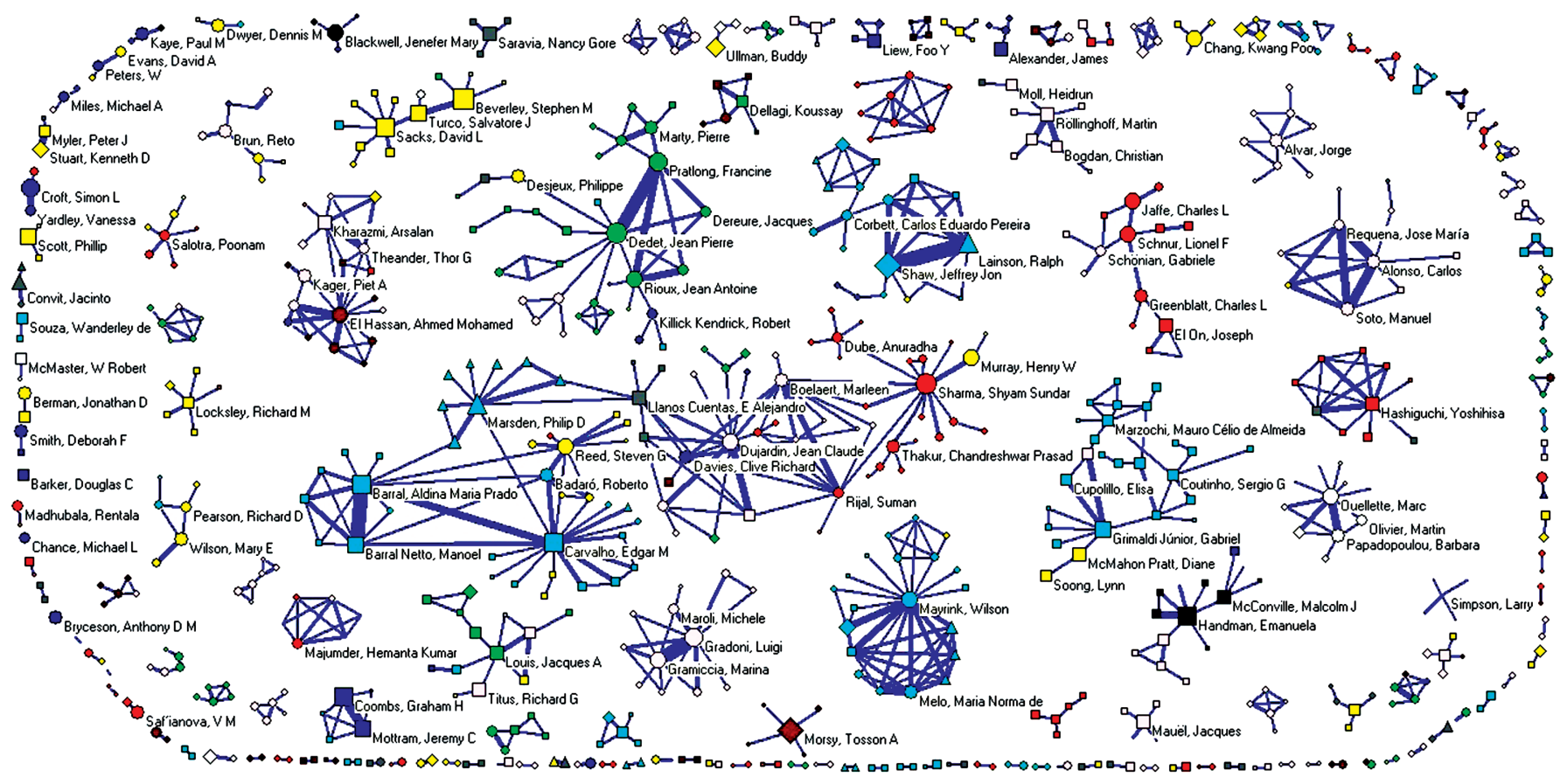

FIGURE 2 - Main research hubs in scientific publications on leishmaniasis ( $>9$ coauthorships). Shapes: ellipse (leishmaniasis visceral), box (leishmaniasis cutaneous), triangle (leishmaniasis mucocutaneous), and diamond (several forms). Colors: blue (Brazil), yellow (USA), green (France), red (India), dark blue (United Kingdom), pink (other European countries), white (Canada), orange (other Near East and Asian countries), brown (African countries), black (Australia), and grey (Peru). The size of the nodes is proportional to the number of papers published and the thickness of the links to the intensity of collaboration (number of coauthored papers). 


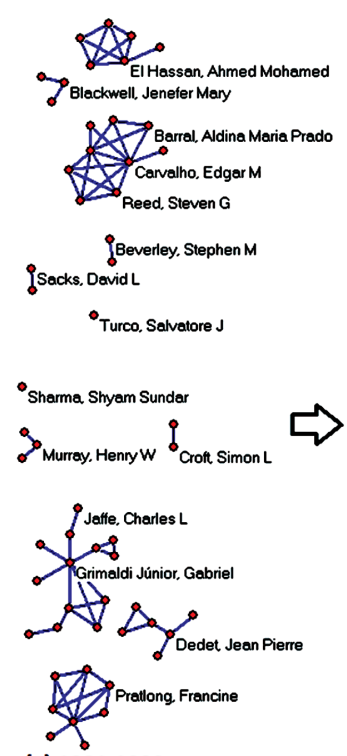

(a) 1981-1990

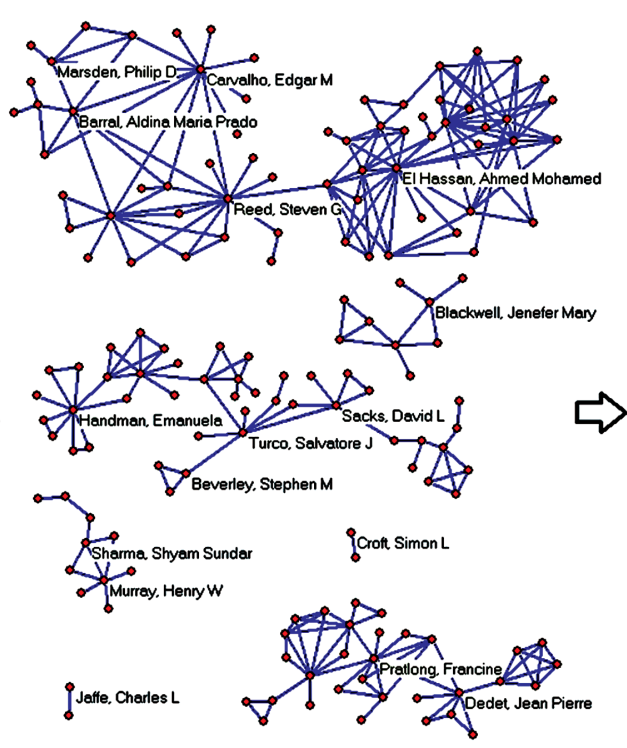

(b) 1991-2000

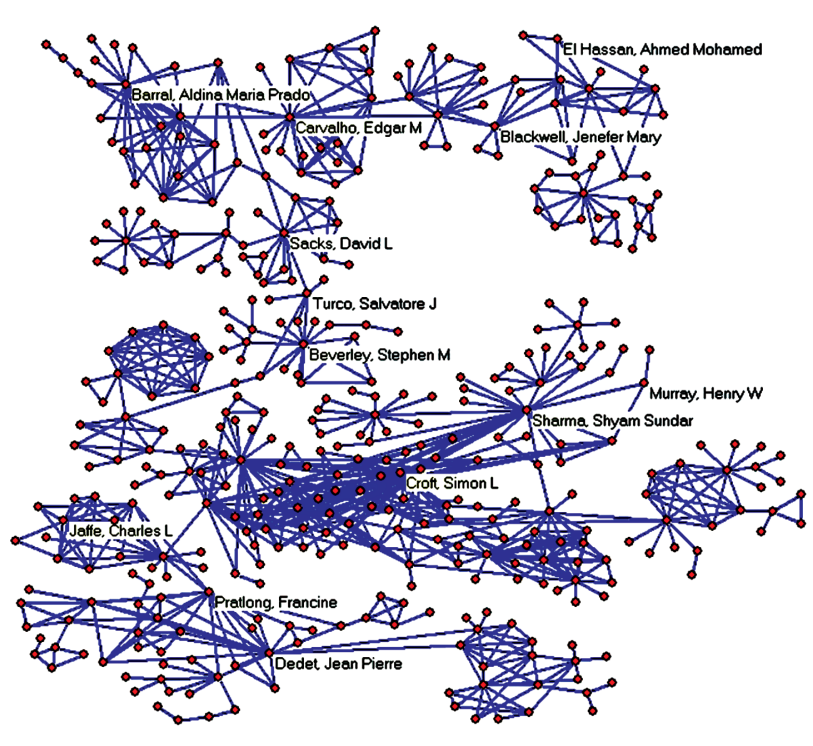

(c) 2001-2010

FIGURE 3 - Evolution by decade of the components integrated in the 2001-2010 period in the giant component.

the analysis on the consolidated research hubs. Over the three decades represented in the figure, an initial group of small components and isolated authors (1981-1990) is progressively transformed; the nodes come together in a process of aggregation or nuclearization around several outstanding authors (who are generally highly productive), resulting in the creation of larger components (1991-2000). This process intensifies in the last decade under study (2001-2010) as the collaborative links multiply, generating a high density of relationships among the different clusters of authors who comprise the main component.

\section{DISCUSSION}

Although all areas of knowledge have experienced noticeable growth in recent years, diverse studies have drawn attention to the even higher rates reported in scientific publications on tropical diseases ${ }^{11}$, particularly leishmaniasis ${ }^{6-8}$. Some reasons may include the impulse shown by WHO and the new interest in neglected diseases through initiatives such as the TDR Programme, the Special Programme for Research and Training in Tropical Diseases started in the mid-1970s, the changes in global knowledge, and some outstanding developments and papers that have raised interest in research on the topic ${ }^{12}$.

The progressive growth in collaboration over the last few decades is one of the defining features of the evolution of science, which helps to explain the increasingly specialized nature of knowledge and the need for an interdisciplinary or multidisciplinary approach to complex problems. This strategy also allows for easier access to technologies and equipment, better management of processes, and a more positive impact with regard to the quality of the results obtained. Keiser and Utzinger ${ }^{11}$ identified a significant increase in the average number of authors per paper in publications on tropical medicine between 1952 and 2002, a rise which has continued to the present day. This general trend also applies to specific tropical diseases, such as Chagas disease ${ }^{13}$. The present study, in addition to confirming this same trend in relation to leishmaniasis, has allowed us to describe other aspects of the scientific collaboration in the field, such as the fact that in last several decades, the number of different people with whom researchers collaborate has significantly increased. Indeed, the average degree tripled from 1971-1980 (3.4) to 2001-2010 (10.6). This trend, together with the fact that the percentage of authors integrated in the largest component has been gradually increasing over time, even as the number of isolates that have not established collaborative links has been decreasing, is a reflection of the higher degree of interconnection observed among scientists and the importance that cooperative research has been acquired over the last few decades; this method is now the principal and defining feature that characterizes the social organization of researchers in their quest to generate new knowledge ${ }^{14}$. The percentage of authors with scientific publications on leishmaniasis and integrated in the largest component throughout the period (79.7\%) is similar to that observed in the coauthorship network observed in the work performed on Chagas disease, where this value was $84.1 \%{ }^{13}$. Even higher figures have been observed in some fields, where up to $95 \%$ of authors are included ${ }^{15}$.

The increase in scientific collaboration has been favored by other factors apart from those already mentioned; the process of globalization has been an important driver of this tendency, facilitating researcher mobility, access to information sources, communication, and the development of Information Technology (IT) tools for cooperative work. These conditions 
have all favored the creation of collaborative links between researchers that are expressed through coauthorships in scientific publications ${ }^{16}$. Inversely, the integration of researchers in the largest component has also resulted in a gradual reduction in the network density, a reflection of the multiplication of research hubs at a global level and the formation of small-world networks, to which the coauthorship networks adapt.

Another of the defining features of the scientific collaboration networks is the fact that stable collaborative relationships can only be maintained with a small number of researchers, whereas most collaborations occur only once (this is the case for $83.1 \%$ of the coauthorships identified in the present study). Unlike the number of total collaborators, this feature is constant throughout the entire period analyzed, with values ranging from $81 \%$ to $92 \%$.

The fact that $47 \%$ of the clusters or groups identified comprise only two authors is concurrent with the value observed by Yu et al ${ }^{15}$ in the coauthorship networks considering coronary heart disease. Although some clusters are more numerous, many of the authors comprising these clusters are rather disconnected from each other, separated by a great number of intermediaries. It is possible to distinguish different subgroups of authors who present a higher degree of cohesion and density in their relationships, suggesting that these nuclei of stable collaborators constitute the customary structures that articulate the scientific research, independently of the sporadic collaborations that exist with other authors.

The diachronic evolution of the coauthorship networks on leishmaniasis coincides with that observed by Lee et al. ${ }^{17}$ in physics; these authors identified three phases: the emergence of small components (nuclearization); their gradual integration into a giant component; and the multiplication of the links in that giant component, generating a dense and complex web of interrelationships. In line with what we have observed in the present study, this process is largely facilitated by the establishment of collaborative links between the most productive authors or between those authors who occupy a role of leadership within their respective components. These researchers bring together a large number of authors, so when they collaborate with each other, they increase the size of the components that they lead. This phenomenon is in line with the preferential attachment model of growth proposed by Barabasi and Albert ${ }^{18}$, in which the nodes with a higher number of relationships have a higher probability of connecting to new nodes.

In addition to graphic representations that facilitate the visualization and analysis of collaboration structures in an area of knowledge, social network analysis can be used to identify key agents in a given field of study, enabling the cohesion or facilitating the connectivity and the information flows in the network. Borgatti ${ }^{19}$ identified two types of key players: those who stand out for their position as agents that bring together a large group of authors, maintaining the cohesion of the network and enabling the interconnection among its members by means of direct links or shortest pathways; and authors who act as a bridge, enabling the interconnection of different parts of the network and impeding its disintegration into separate and smaller components. In the main component of the leishmaniasis network, Edgar M. Carvalho, Aldina María Prado Barral, Philip D. Marsden, Steven G. Reed, Jean Claude Dujardin, and Shyam Sundar Sharma would fit into the first group, whereas E. Alejandro Llanos Cuentas would be the paradigmatic example of the second group.

The clusters or research groups identified have a marked national character, and they generally investigate a single clinical strain of leishmaniasis, although occasionally some authors or research groups in the same country study different clinical forms of the disease. The collaboration among authors who investigate different clinical forms of leishmaniasis is limited, presumably because the pathologies require different therapeutic responses. However, some collaborative relationships between authors with different leishmaniasis specialties do exist, possibly due to migration of the vector or the presence of animal reservoirs, which has led to an extension of studies on this disease to places where few or no cases have been reported historically ${ }^{20}$. These collaborations may also focus on aspects such as epidemiology, the vector or the reservoir, giving rise to collaborations between entomologists, biologists, medical doctors, epidemiologists, and specialists in public health, who may specialize in one or various forms of leishmaniasis.

The observation by Al-Mutawaket et al. ${ }^{6}$, who observed an absolute dominion of the USA in terms of international collaboration, can be qualified based on the results obtained in our analysis of the coauthorship networks. Although we confirm that the USA boasts more international ties, Brazil is shown to play a leadership role in the research on the topic, with three outstanding hubs in the center of the network, dominated by Brazilian authors, as well as a notable presence in the largest component. The preeminence of these groups and the presence of numerous Brazilian investigators in the identified research groups stems from the fact that leishmaniasis is an important public health problem in Brazil, where incidence of the disease in all of its forms is higher than in the remainder of the continent. Thus, Brazil is developing its research potential in the field, making leishmaniasis - as well as other neglected diseases -a priority on the national health research agenda ${ }^{21-23}$.

With regard to the collaboration between researchers from different countries, several factors come into play in the pronounced degree of collaboration between the USA and Brazil. In addition to the development of the scientific systems in both countries, there is the geographic proximity between them (relative to other countries, such as India) and the institutional links developed through academic exchange programs or grants, as researchers who return from the USA or Europe generally maintain their collaborative ties or initiate other common projects with their colleagues from abroad ${ }^{24}$. To understand the collaborations among researchers, some authors have drawn attention to the importance of homophily, that is, sharing certain attributes, such as age, sex, academic or professional rank, or amount of similar experience ${ }^{25}$. Previous experiences also shape relationships; decisive factors that contribute to stable collaborative links include compatibility of working styles, the creation of a trustful working climate, and the existence of common interests ${ }^{16}$. 
The collaboration among European countries is favored by several circumstances, including the incidence of scientific policies that aim to foster research in all areas of knowledge, the traditional leadership of these countries - together with the USA - at a scientific level, and their interest in protecting European populations from tropical diseases whose emergence on the continent has been precipitated by migratory and touristic movements. Interest in research on leishmaniasis has also been attributed to the fact that it constitutes and excellent parasitic model ${ }^{6}$.

The fact that more than $90 \%$ of all cases of visceral leishmaniasis are concentrated in six countries (India, Bangladesh, Sudan, South Sudan, Brazil, and Ethiopia) ${ }^{1}$ contrasts with the limited development of research on the topic in these countries, with the exceptions of India and Brazil, as shown by the results of our analysis of research groups. In addition to this scarce research, Keiser and Utzinger ${ }^{11}$ have signaled the limited presence of authors from developing countries in positions of leadership and prestige in scientific publications in the field of tropical medicine (first and last signature on author lists), a situation which would have reflected the end of scientific colonization, a situation in which researchers in wealthy countries and those on the vanguard of scientific development were the ones to set priorities and lines of research for developing countries, in favor of a situation of scientific subordination. Although collaboration with more developed countries can constitute an effective mechanism to stimulate and foster research in less developed nations (a fact which has given rise over the years to numerous initiatives to promote North-South collaborations) $)^{3}$, the ultimate aim to avoid this type of subordination should be to create stable research structures in developing countries and to develop South-South collaborations through the joint development of research initiatives, which are particularly important in the case of health problems that directly affect these countries ${ }^{21}$. Regional reference centers and existing collaboration networks have an important role to play in promoting research, establishing collaborative alliances between other emerging countries, and integrating researchers from these countries into international research networks ${ }^{26}$.

Attention has also been drawn to the importance of developing initiatives that attract private interests, particularly pharmaceutical companies, as the lack of commercial interest from this sector constitutes one of the main barriers to the development of drugs to treat neglected diseases ${ }^{23,26}$. In this respect, the formation of multidisciplinary research networks and the establishment of collaborative alliances between the public sector and industry should be fostered ${ }^{26}$.

Finally, other authors have also noted that to tackle neglected diseases, it is essential to consider the economic, political, social, cultural, and environmental context in which they appear, promoting research in social science disciplines, such as anthropology, health economics, medical sociology, social policy, and social epidemiology, among others. In disease control, contributions from these fields can be as important as biomedical research focused on the study of the infectious agent and the corresponding therapeutic response ${ }^{27}$.

Any study that aims to analyze collaboration through coauthorships in scientific publications must assume certain limitations given that the fact that two authors signing a paper does not necessarily mean that a real collaboration has been established between them or that all collaborations have been faithfully reflected in the author list. Moreover, other phenomena or customs practiced at the time of determining the author list might distort these analyses, such as unjustified hyperauthorship ${ }^{28}$. In addition, other phenomena, such as the high mobility of researchers, may affect the results presented and should be considered. However, this type of study can be of great use, offering an approach to better understand the markedly cooperative organizational and social context in which scientific knowledge is generated.

\section{CONFLICT OF INTEREST}

The authors declare that there is no conflict of interest.

\section{REFERENCES}

1. Alvar J, Vélez, ID, Bern C, Herrero M, Desjeux P, Cano J, et al. Leishmaniasis worldwide and global estimates of its incidence. PLoS One 2012; 7:e35671.

2. Antinori S, Schifanella L, Corbellino M. Leishmaniasis: new insights from an old and neglected disease. Eur J Clin Microbiol Infect Dis 2012; 31:109-118.

3. Keiser J, Utzinger J, Tanner M, Singer BH. Representation of authors and editors from countries with different human development indexes in the leading literature on tropical medicine: survey of current evidence. BMJ 2004; 328:1229-1232.

4. Newman R. Parasitology research in Europe. Lab Times 2009; 3:38-40.

5. Smith DR. Citation indexing and the development of academic journals in tropical medicine. Mem Inst Oswaldo Cruz 2008; 103:310-312.

6. Al-Mutawakel K, Scutaru C, Shami A, Sakr M, Groneberg DA, Quarcoo D. Scientometric analysis of the world-wide research efforts concerning leishmaniasis. Parasit Vectors 2010; 3:14.

7. Fallah E, Biglu MH. Scientific production of leishmaniasis in PubMed: Impact of Iranian institutes. Int J Adv Pharm Sci 2011; 2:1-7.

8. Ramos JM, González Alcaide G, Bolaños Pizarro M. Bibliometric analysis of Leishmaniasis research in Medline (1945-2010). Parasit Vectors 2013; 6:55.

9. Persson OD, Danell R, Schneider JW. How to use Bibexcel for various types of bibliometric analysis. In: Åström F, Danell R, Larsen B, Schneider J, editors. Celebrating scholarly communication studies: a Festschrift for Olle Persson at his 60th Birthday. Belgium: International Society for Scientometrics and Informetrics; 2009. p. 9-24.

10. Batagelj V, Mrvar A. Pajek: analysis and visualization of large networks. In: Jünger M, Mutzel P, editors. Graph Drawing Software. Berlin: Springer; 2003. p. 77-103.

11. Keiser J, Utzinger J. Trends in the core literature on tropical medicine: a bibliometric analysis from 1952-2002. Scientometrics 2005; 62:351-365.

12. Chinnock P. Leishmaniasis attracts increased attention from researchers [Internet]. Tropical Diseases Research to Foster Innovation \& Knowledge Application (TropIKA.net); 2010; [Cited 2013 Nov 21]. Available at: http://www.tropika.net/svc/research/Chinnock-20100414-ResearchLeish/.

13. González Alcaide G, Park J, Huamaní C, Gascón J, Ramos Rincón JM. Scientific authorships and collaboration network analysis on Chagas disease: papers indexed in PubMed (1940-2009). Rev Inst Med Trop Sao Paulo 2012; 54:219-228.

14. Uddin S, Hossain L, Abbasi A, Rasmussen K. Trend and efficiency analysis of co-authorship network. Scientometrics 2012; 90:687-699. 
15. Yu Q, Shao H, He P, Duan Z. World scientific collaboration in coronary heart disease research. Int J Cardiol 2013; 167:631-639.

16. Hara N, Solomon P, Kim SL, Sonnenwald DH. An emerging view of scientific collaboration: scientists' perspectives on collaboration and factors that impact collaboration. J Am Soc Inf Sci Technol 2003; 54: 952-965.

17. Lee D, Goh KI, Kahng B, Kim D. Complete trails of coauthorship network evolution. Phys Rev E 2010; 82:026112.

18. Barabasi AL, Albert R. Emergence of scaling in random networks. Science 1999; 286:509-512.

19. Borgatti SP. Identifying sets of key players in a social network. Comput Math Organ Theory 2006; 12:21-24.

20. McDowell MA, Rafati S, Ramalho-Ortigao M, Ben Salah A. Leishmaniasis: Middle East and North Africa Research and Development Priorities. PLoS Negl Trop Dis 2011; 5:e1219.

21. Dujardin JC, Herrera S, Rosario V, Arevalo J, Boelaert M, Carrasco HJ, et al. Research priorities for neglected infectious diseases in Latin America and the Caribbean Region. PLoS Negl Trop Dis 2010; 4:e780.

22. Morel CM, Serruya SJ, Penna GO, Guimarães R. Co-authorship network analysis: a powerful tool for strategic planning of research, development and capacity building programs on neglected diseases. PLoS Negl Trop Dis 2009; 3:e501.

23. Vasconcellos AG, Morel CM. Enabling policy planning and innovation management through patent information and co-authorship network analyses: a study of Tuberculosis in Brazil. PLoS One 2012; 7:e45569.

24. Gotuzzo E, González E, Verdonck K. Researchers training in the context of the collaborative projects: experiences of Instituto de Medicina Tropical "Alexander von Humbolt", Universidad Peruana Cayetano Heredia. Rev Peru Med Exp Salud Publica 2010; 27:419-427.

25. Long JC, Cunningham FC, Braithwaite J. Network structure and the role of key players in a translational cancer research network: a study protocol. BMJ Open 2012; 2:e001434.

26. Jakobsen PH, Wang MW, Nwaka S. Innovative partnerships for drug discovery against neglected diseases. PLoS Negl Trop Dis 2011; 5:e1221.

27. Allotey P, Reidpath DD, Pokhrel S. Social sciences research in neglected tropical diseases 1: the ongoing neglect in the neglected tropical diseases. Health Res Policy Syst 2010; 8:32.

28. Cronin B. Hyperauthorship: A postmodern perversion or evidence of a structural shift in scholarly communication practices? J Am Soc Inf Sci Technol 2001; 52:558-569. 